\title{
MHD ON A CYLINDER IMPLANTED IN A POROUS MEDIA OF MICROPOLAR FLUID
}

\author{
MADASU KRISHNA PRASAD*, TINA BUCHA \\ Department of Mathematics, National Institute of Technology, \\ Raipur-492010, Chhattisgarh, India \\ [Received: 05 July 2019. Accepted: 05 October 2020] \\ doi: 10.7546/JTAM.50.20.04.01
}

\begin{abstract}
This article handles MHD flow of micropolar fluid through a rigid cylindrical particle implanted in a porous media. Flow is considered to be uniform at a distance away from the cylinder. A uniform magnetic field is applied perpendicular to the flow direction. Motion in porous media is governed by Brinkman's equation. At the surface of cylinder, we assume no-slip of velocity components and no-spin of microrotational component. Exact solution for the problem is reported. Influence of Hartmann number, permeability and micropolarity parameters on the coefficient of drag and tangential velocity are discussed numerically. In deduction, various results of flow past impenetrable cylinder are obtained which are in good agreement with earlier published works.
\end{abstract}

KEY WORDS: micropolar fluid, magnetic field, Brinkman's model, drag force.

\section{INTRODUCTION}

Over recent years, an increased attention for the study of fluid flow under the influence of magnetic field in different physical circumstances has been widely considered. Magnetic field influences various natural and manmade flows. Magnetohydrodynamic (MHD) is the field of science, which includes studying the motion of electrically conducting fluids. Application of MHD can be found in diverging fields like astrophysics, geophysics, industrial science, biomedical science etc.

In literature, flow problems considering classical Newtonian fluid past a particle implanted in porous media was investigated by many authors. Darcy's law [1] and Brinkman's model [2] has been extensively used for modelling flow past porous medium. Speilman and Goren [3] considered a model for studying fluid flow past a circular cylinder. Pop and Cheng [4] examined the fluid flow past a cylindrical particle inserted in a media consisting of porous structure by using Brinkman's equation. Feng et al. [5] discussed the motion of a sphere close to a planar confining boundaries using Brinkman model. Wang [6] handled the problem of the viscous fluid flow over a particle of different geometry like sphere, a circular cylinder and solid inclusion in a porous media governed by Darcy-Brinkman's equation. Satya et al. [7] examined the

\footnotetext{
${ }^{*}$ Corresponding author e-mail: madaspra.maths@ nitrr.ac.in
} 
creeping flow past an aggregate of porous cylindrical particle. Further, Leontev [8] investigated the flow through a spherical and a cylindrical particle using Navier slip boundary condition.

Many researchers for characterizing the behaviour of real fluid have modelled wide range of problems considering several fluids. Recently, fluids with microstructure are focused with utmost importance due to its need to model various engineering processes, which fails to be properly described by the classical Newtonian fluid. Some of their applications include blood flow, lubrication, colloidal fluids, complex biological structures, porous media, flow in capillaries, microchannels, suspension fluids etc. Eringen $[9,10]$ illuminated the basic concept behind the micropolar fluid. Fluids with microstructure consist of microelements possessing dumbbell shape, which have a tendency to rotate with their own spin and micro rotation. Earlier study by Rao and Rao [11] shows the dependence of drag on micropolarity parameter and it was observed that the drag force exerted on the sphere for micropolar fluid flow to be higher as compared with Newtonian fluid. Hayakawa [12] obtained drag acted on the stationary sphere and cylinder due to micropolar fluid flow. Hamdan and Kamel [13] handled unsteady incompressible micropolar flow through isotropic porous sediments using intrinsic volume-averaging techniques. Later, Srinivasacharya and Prasad [14] discussed the micropolar flow past a cylinder and sphere implanted in a saturated porous media. They showed the influence of micropolarity on particles and found the drag to be increasing with enhancing micropolarity. Subsequently, Jaiswal [15] focused on considering a liquid sphere of non-Newtonian fluid embedded in a Stokes incompressible micropolar fluid saturated porous medium. $\mathrm{Kr}$ ishnan et al. [16] studied resisting force acted on a fluid sphere with solid core embedded in a porous media filled with micropolar fluid. They have considered Brinkman's equation for studying motion in porous region and have obtained the drag force acting on the fluid sphere. In recent article by Khanukaeva et al. [17, 18] motion of micropolar fluid through a cylinder containing an impervious core in a direction parallel and perpendicular to the axis of cylindrical cells in executed. They considered two varying boundary conditions for micro polar fluid and also found an expression for hydrodynamic permeability.

Study of flow under MHD has welcomed many researchers [19,20]. Raghava Rao and Sekhar [21] reported quantitative study of MHD flow past a cylindrical particle. Geindreau and Aurlialt [22] investigated the tensorial filtration law in porous media under MHD effect by using Darcy's law. Verma and Datta [23] reported effect of MHD on channel consisting of varying viscosity. Tiwari et al. [24] worked on finding MHD effect on the hydrodynamic permeability for flow through a porous cylinder with solid core by employing cell model technique. Jayalakshmamma et al. [25] discussed MHD fluid flow on solid core covered by a cylindrical region. Verma and 
Gupta [26] obtained the solution of the MHD flow of fluid in cylindrical channel partly filled with porous media in analytical approach. Saad [27] tackled MHD on the motion of a sphere and a cylinder with porous structure. He obtained the expression for Kozeny constant and had compared the results for particles with varying geometry. Subsequently, Ansari et al. [28] discussed the motion of fluid through a sphere implanted in porous media under the action of magnetic field. In the very recent articles by Prasad and Bucha [29-32], the investigation of the impact of applied transverse magnetic field on the flow of Newtonian fluid past particles with different geometrical shapes is reported. Inspired by the above work, we are interested in examining the problem considered by Prasad and Srinivasacharya [14] under transverse uniform magnetic field. The current study of particles implanted in porous media is very fundamental in the study of fluid and heat transfer and are useful while dealing with transport phenomena, like heat, mass, and momentum transfer in porous media. Applying the magnetic field on such flow can serve the purpose of controlling the heat transfer rate for various thermal engineering systems [33].

In the present scenario, we are examining MHD effect on a cylinder implanted in porous media filled with micropolar fluid. We focus on finding the expression for stream function, microrotation and pressure. Drag exerted on the embedded cylinder is reported. Graphs are used to depict the variation of coefficient of drag and tangential velocity for varying values of Hartmann number, permeability parameter, micropolarity parameter and radius of the cylinder.

\section{Problem Formulation}

A steady incompressible flow of micropolar fluid past an impervious cylindrical particle bearing radius $r=a$ implanted in porous medium under applied magnetic field is considered (see Fig. 1). Consider $(r, \theta, z)$ to denote a cylindrical polar coordinate system and $\left(\vec{e}_{r}, \vec{e}_{\theta}, \vec{e}_{z}\right)$ as unit basis vectors. A uniform velocity $U$ is assumed far away from the sphere along the axis of symmetry $\theta=0$. Magnetic field is chosen in a direction perpendicular to the flow, such that $\vec{H}=H_{0} \vec{e}_{r}$. It is assumed that no external electric field is applied and the induced magnetic field is also neglected.

Lorentz force $\vec{F}$ due to applied magnetic field is defined as

$$
\vec{F}=\mu_{h}^{2} \sigma(\vec{q} \times \vec{H}) \times \vec{H}
$$

with $\vec{H}$ as the magnetic field intensity, $\mu_{h}$ to be the magnetic permeability and $\sigma$ as the electric conductivity.

Lorentz force balances pressure and viscous stresses to obtain modified Brinkman's equation. Equations governing micropolar fluid in the porous medium under mag- 


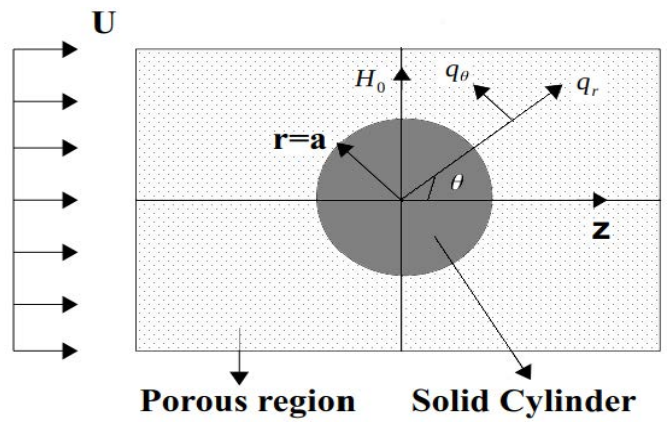

Fig. 1: Sketch of micropolar flow past cylinder with MHD effect.

netic effect is given as $[14,27]$

(1) $\quad \nabla \cdot \vec{q}=0$

(2) $\nabla p+\left(\frac{\mu+\kappa_{p}}{k}\right) \vec{q}+\left(\mu+\kappa_{p}\right) \nabla \times \nabla \times \vec{q}-\kappa_{p} \nabla \times \vec{\nu}$

$$
-\frac{\mu_{h}^{2} \sigma}{\epsilon}(\vec{q} \times \vec{H}) \times \vec{H}=0,
$$

$$
\kappa_{p} \nabla \times \vec{q}-2 \kappa_{p} \vec{\nu}-\gamma_{0} \nabla \times \nabla \times \vec{\nu}+\left(\alpha_{0}+\beta_{0}+\gamma_{0}\right) \nabla(\nabla \cdot \vec{\nu})=0,
$$

where $\vec{q}, k, \epsilon, \vec{\nu}, p, \mu, \kappa_{p}$ are the velocity vector, permeability parameter, porosity of the porous region, microrotation vector, pressure, coefficient of viscosity for the classical viscous fluid and rotational viscosity coefficient, respectively. $\alpha_{0}, \beta_{0}, \gamma_{0}$ as the coefficients of gyroviscosity for micropolar fluid.

We denote

$$
\begin{aligned}
& \tau_{i j}=-p \delta_{i j}+\mu\left(\vec{q}_{i, j}+\vec{q}_{j, i}\right)+\kappa_{p}\left(\vec{q}_{j, i}-\epsilon_{i j m} \vec{\nu}_{m}\right), \\
& m_{i j}=\alpha_{0} \vec{\nu}_{m, m} \delta_{i j}+\beta_{0} \vec{\nu}_{i, j}+\gamma_{0} \vec{\nu}_{j, i}
\end{aligned}
$$

as the stress tensor and the couple stress tensor respectively. $\delta_{i j}, \epsilon_{i j m}$ are the Kronecker delta, the alternating tensor, and the comma denotes the covariant differentiation.

Fluid flow is supposed to be axially symmetric, therefore all the physical quantities employed in the flow are independent of $z$.

So, we considered velocity and microrotational components as

$$
\begin{gathered}
\vec{q}=q_{r}(r, \theta) \vec{e}_{r}+q_{\theta}(r, \theta) \vec{e}_{\theta}, \\
\vec{\nu}=\nu(r, \theta) \vec{e}_{z} .
\end{gathered}
$$


We choose non-dimensional variables, for converting the governing equations in dimensionless form as

(8) $\quad r=a \tilde{r}, \quad \vec{q}=U \tilde{\vec{q}}, \quad \nabla=\frac{1}{a} \tilde{\nabla}, \quad p=\frac{\mu}{a} U \tilde{p}, \quad \vec{H}=H_{0} \tilde{\vec{H}}, \quad \vec{\nu}=\frac{U}{a} \tilde{\vec{\nu}}$.

Using Eq. (8) in Eqs. (2) and (3), and dropping tildes, we obtain

(9) $\nabla p+\eta^{2}(1+\chi) \vec{q}+(1+\chi) \nabla \times \nabla \times \vec{q}-\chi \nabla \times \vec{\nu}-\alpha^{2}(\vec{q} \times \vec{H}) \times \vec{H}=0$,

$$
\nabla \times \vec{q}-2 \vec{\nu}-n^{-2} \nabla \times \nabla \times \vec{\nu}+s^{-2} \nabla(\nabla \cdot \vec{\nu})=0 .
$$

The dimensionless number $\chi=\kappa_{p} / \mu$ is the ratio of the coefficient of rotational viscosity to the classical viscosity known as a micropolarity parameter. It enables to measure the deviation of micropolar fluid flow from that of Newtonian fluid. $\eta^{-2}=$ $\frac{k}{a^{2}}$ is a dimensionless permeability parameter which characterizes the specifics of the filtration part of the flow. $\alpha=\sqrt{\frac{\sigma \mu_{h}^{2} H_{0}^{2} a^{2}}{\epsilon \mu}}$ is the Hartmann number which signifies the magnetic effect on the flow. It provides a measure of the relative importance of drag forces resulting from magnetic induction and viscous forces. $n^{2}=a^{2} \kappa_{p} / \gamma_{0}$, $s^{2}=\frac{a^{2} \kappa_{p}}{\left(\alpha_{0}+\beta_{0}+\gamma_{0}\right)}$ denote the parameters that represent the relation between gyro viscosities and rotational viscosity.

For our convenience, we introduce stream function $\psi$ related to velocity components as

$$
q_{r}=-\frac{1}{r} \frac{\partial \psi}{\partial \theta}, \quad q_{\theta}=\frac{\partial \psi}{\partial r} .
$$

Substituting Eq. (11) in Eq. (9), we obtain

$$
\begin{aligned}
\frac{\partial p}{\partial r} & =\frac{1}{r}\left[\chi \frac{\partial \nu}{\partial \theta}+\left((1+\chi) \eta^{2}+\alpha^{2}\right) \frac{\partial \psi}{\partial \theta}-(1+\chi) \frac{\partial}{\partial \theta} \nabla^{2} \psi\right], \\
\frac{1}{r} \frac{\partial p}{\partial \theta} & =-\left[\chi \frac{\partial \nu}{\partial r}+\left((1+\chi) \eta^{2}+\alpha^{2}\right) \frac{\partial \psi}{\partial r}-(1+\chi) \frac{\partial}{\partial r} \nabla^{2} \psi\right] .
\end{aligned}
$$

Eliminating pressure term from the above equations, we obtain

$$
\nabla^{2} \nu=\frac{(1+\chi)}{\chi}\left[\nabla^{4} \psi-\delta \nabla^{2} \psi\right]
$$

where $\nabla^{2}=\frac{\partial^{2}}{\partial r^{2}}+\frac{1}{r} \frac{\partial}{\partial r}+\frac{1}{r^{2}} \frac{\partial^{2}}{\partial \theta^{2}}$ is the Laplacian operator and

$$
\delta=\eta^{2}+\frac{\alpha^{2}}{1+\chi} .
$$


Again using Eq. (11) in Eq. (10), we obtain

$$
\nabla^{2} \psi-2 \nu+n^{-2} \nabla^{2} \nu=0 .
$$

Using Eq. (12) and Eq. (13), we have

$$
\nu=\frac{1}{2}\left[L \nabla^{4} \psi+(1-L \delta) \nabla^{2} \psi\right],
$$

where $L=(1+\chi) /\left(\chi n^{2}\right)$.

From Eq. (12) and (14), we get

$$
\nabla^{2}\left(\nabla^{2}-\lambda_{1}^{2}\right)\left(\nabla^{2}-\lambda_{2}^{2}\right) \psi=0,
$$

where

$$
\lambda_{1}^{2}+\lambda_{2}^{2}=2 n^{2}-L^{-1}+\delta, \quad \lambda_{1}^{2} \lambda_{2}^{2}=2 n^{2} \delta .
$$

Therefore,

$$
\lambda_{1}=\sqrt{\frac{\delta+2 n^{2}-L^{-1}+\sqrt{\left(\delta+2 n^{2}-L^{-1}\right)^{2}-8 n^{2} \delta}}{2}}, \quad \lambda_{2}=\sqrt{\frac{2 n^{2} \delta}{\lambda_{1}^{2}}} .
$$

In absence of MHD effect, i.e. $\alpha=0$, the above values of $\lambda_{1}^{2}+\lambda_{2}^{2}, \lambda_{1}^{2} \lambda_{2}^{2}$ are identical with the work of Prasad and Srinivasacharya [14].

\section{BOUNDARY CONDITIONS}

Boundary conditions used at the surface on $r=1$ of the cylinder for finding the flow velocities and microrotational components and pressure are as follow:

(i) No slip condition [14]:

$$
q_{r}=0, \quad q_{\theta}=0,
$$

(ii) No spin condition [14]:

$$
\nu=0
$$

\section{Solution of the Problem}

Expression for the solution of Eq. (15) in terms of stream function is

$$
\begin{aligned}
\psi & =\left[r+A \frac{1}{r}+B K_{1}\left(\lambda_{1} r\right)+C K_{1}\left(\lambda_{2} r\right)\right] \sin \theta, \\
\nu & =\frac{1}{2}\left[B \zeta_{1} K_{1}\left(\lambda_{1} r\right)+C \zeta_{2} K_{1}\left(\lambda_{2} r\right)\right] \sin \theta,
\end{aligned}
$$


where $K_{1}\left(\lambda_{1} r\right), K_{1}\left(\lambda_{2} r\right)$ denote the modified Bessel functions of second kind with order 1 , and

$$
\begin{aligned}
& \zeta_{1}=[1-L \delta] \lambda_{1}^{2}+L \lambda_{1}^{4}, \\
& \zeta_{2}=[1-L \delta] \lambda_{2}^{2}+L \lambda_{2}^{4} .
\end{aligned}
$$

The expression for pressure is

$$
p=\left[\eta^{2}(1+\chi)+\alpha^{2}\right](r-A / r) \cos \theta .
$$

By substituting Eqs. (18) to (22) in the boundary conditions we have the following algebraic equations as:

$$
\begin{aligned}
& 1+A+K_{1}\left(\lambda_{1}\right) B+K_{1}\left(\lambda_{2}\right) C=0, \\
& 1-A-\left(K_{1}\left(\lambda_{1}\right)+\lambda_{1} K_{0}\left(\lambda_{1}\right)\right) B-\left(K_{1}\left(\lambda_{2}\right)+\lambda_{2} K_{0}\left(\lambda_{2}\right)\right) C=0, \\
& \zeta_{1} K_{1}\left(\lambda_{1}\right) B+\zeta_{2} K_{1}\left(\lambda_{2}\right) C=0 .
\end{aligned}
$$

Solving Eqs. (23) to (25), the obtained values of $A, B, C$ are

$$
\begin{aligned}
A & =-\left[1+\frac{2 K_{1}\left(\lambda_{1}\right) K_{1}\left(\lambda_{2}\right)\left(\lambda_{1}^{-1} \lambda_{2} \Omega_{1}-\lambda_{1} \lambda_{2}^{-1} \Omega_{2}\right)}{\Delta}\right], \\
B & =\frac{2 K_{1}\left(\lambda_{2}\right) \lambda_{2} \Omega_{1}}{\lambda_{1} \Delta} \\
C & =-\frac{2 K_{1}\left(\lambda_{1}\right) \lambda_{1} \Omega_{2}}{\lambda_{2} \Delta}
\end{aligned}
$$

where

$$
\begin{aligned}
& \Omega_{1}=\left(\lambda_{2}^{2}+\delta_{1}\right) \chi+\lambda_{2}^{2}-\delta_{2}, \\
& \Omega_{2}=\left(\lambda_{1}^{2}+\delta_{1}\right) \chi+\lambda_{1}^{2}-\delta_{2}, \\
& \Delta=\lambda_{2} K_{0}\left(\lambda_{1}\right) K_{1}\left(\lambda_{2}\right) \Omega_{1}-\lambda_{1} K_{1}\left(\lambda_{1}\right) K_{0}\left(\lambda_{2}\right) \Omega_{2}, \\
& \delta_{1}=n^{2}-\eta^{2}, \\
& \delta_{2}=\eta^{2}+\alpha^{2} .
\end{aligned}
$$

\section{DRAG ACTING ON THE CYLINDER}

Drag exerted on the cylindrical particle under transverse magnetic field can be evaluated by using the formula $[7,14]$ :

$$
F_{D}=\left.\int_{0}^{2 \pi} r\left(\tau_{r r} \cos \theta-\tau_{r \theta} \sin \theta\right)\right|_{r=1} d \theta=-\pi \mu U F
$$


where

$$
\begin{aligned}
F=(2+\chi)\left[\frac{(1+\chi)}{(2+\chi)} \delta(1-A)+\right. & \frac{\lambda_{1}^{2}}{2}\left(1+\frac{(1+\chi)}{(2+\chi) n^{2}}\left(\delta-\lambda_{1}^{2}\right)\right) K_{1}\left(\lambda_{1}\right) B \\
+ & \left.\frac{\lambda_{2}^{2}}{2}\left(1+\frac{(1+\chi)}{(2+\chi) n^{2}}\left(\delta-\lambda_{2}^{2}\right)\right) K_{1}\left(\lambda_{2}\right) C\right] .
\end{aligned}
$$

Substituting the values of $A, B$ and $C$ from Eqs. (26) to (28) in Eq. (29), the required expression of drag in simplified form is

$$
\begin{aligned}
& F_{D}=-2 \pi \mu U\left[K_{1}\left(\lambda_{1}\right) K_{1}\left(\lambda_{2}\right)\left\{\lambda_{1}^{-1} \lambda_{2} \Delta_{4}-\lambda_{1} \lambda_{2}^{-1} \Delta_{3}\right\}\right. \\
& \left.+\lambda_{2} K_{0}\left(\lambda_{1}\right) K_{1}\left(\lambda_{2}\right) \Delta_{1}+\lambda_{1} K_{1}\left(\lambda_{1}\right) K_{0}\left(\lambda_{2}\right) \Delta_{2}\right] / \Delta
\end{aligned}
$$

where

$$
\begin{aligned}
& \Delta_{1}=\eta^{2}\left(\lambda_{2}^{4}+\delta_{1}\right) \chi^{2}+\left(\delta_{4} \lambda_{2}^{2}-\delta_{5}\right) \chi+\delta_{2} \lambda_{2}^{2}-\delta_{6}, \\
& \Delta_{2}=-\eta^{2}\left(\lambda_{1}^{2}+\delta_{1}\right) \chi^{2}-\left(\delta_{4} \lambda_{1}^{2}-\delta_{5}\right) \chi-\delta_{2} \lambda_{1}^{2}+\delta_{6}, \\
& \Delta_{3}=\eta^{2}\left(\lambda_{1}^{2}-\delta_{1}\right) \chi^{2}+\left(\delta_{4} \lambda_{1}^{2}-\delta_{5}\right) \chi+\delta_{2} \lambda_{1}^{2}-\delta_{6}, \\
& \Delta_{4}=\left(\delta_{1} \eta^{2}+\delta_{7}\right) \chi^{2}+\left(\delta_{8}-\delta_{5}\right) \chi-\delta_{6}+\delta_{9}, \\
& \delta_{3}=n^{2}-2 \alpha^{2}, \\
& \delta_{4}=2 \eta^{2}+\alpha^{2}, \\
& \delta_{5}=2 \eta^{4}-\delta_{3} \eta^{2}-n^{2} \alpha^{2}, \\
& \delta_{6}=\eta^{4}+\alpha^{2} \delta_{4}, \\
& \delta_{7}=\left(\lambda_{1}^{2}+\eta^{2}\right) \lambda_{2}^{2}-\lambda_{1}^{4}, \\
& \delta_{8}=\left(2 \lambda_{1}^{2}+\delta_{4}\right) \lambda_{2}^{2}-2 \lambda_{1}^{4}, \\
& \delta_{9}=\left(\lambda_{1}^{2}+\delta_{2}\right) \lambda_{2}^{2}-\lambda_{1}^{4} .
\end{aligned}
$$

If $\alpha=0$ and $\chi=0$ in the expression of $\lambda_{1}$ and $\lambda_{2}$, we found $\lambda_{1}=\eta$ and $\lambda_{2}=0$.

Substituting these values in Eq. (30), the drag acting on a cylinder embedded in Newtonian fluid in absence of magnetic field is obtained as

$$
F_{D}=-2 \pi \mu U \eta^{2}\left[1+2 K_{1}(\eta)\left(\eta K_{1}(\eta)\right)^{-1}\right] .
$$

It agrees with the results of Spielman and Goren [3] and Wang [6]. 


\section{GRAPHICAL REPRESENTATION AND DISCUSSION}

Impact of Hartmann number $\alpha$, micropolarity parameters $\chi$ and permeability $k_{1}=$ $\eta^{-2}$, on the coefficient of drag $D_{N}=\frac{-F_{D}}{\frac{1}{2} \rho U^{2} 2 a}=-\frac{2 \pi F}{R_{e}}$ are examined graphically in Fig. 2 and Fig. 3 respectively. From Fig. 2 it is seen that drag is a monotonically increasing function of Hartmann number. The reason behind the same is the presence of magnetic forces which generates a retarding force known as Lorentz force. This force increases with the increasing Hartmann number and therefore suppress the rate of fluid flow and hence increase in the resistance to the flow, i.e., drag is observed. Overall, MHD effect seems to have a significant role in enhancing the drag force. Figure 3 depicts that with increasing micropolarity effect, drag is also found to be increasing. The curve indicating $\chi=0$ shows the drag acting on the cylinder due to Newtonian fluid. This curve is situated at the lowest among other non-zero micropolarity parameter which clarifies that, the drag force acting on Newtonian fluid is comparatively lower than the drag due to micropolar fluid.

The expression for tangential velocity can be computed from Eqs. (11) and (18) and is given as

$q_{\theta}=\left[1-A \frac{1}{r^{2}}-B\left(\lambda_{1} K_{0}\left(\lambda_{1} r\right)+\frac{1}{r} K_{1}\left(\lambda_{1} r\right)\right)-C\left(\lambda_{2} K_{0}\left(\lambda_{2} r\right)+\frac{1}{r} K_{1}\left(\lambda_{2} r\right)\right)\right] \sin \theta$.

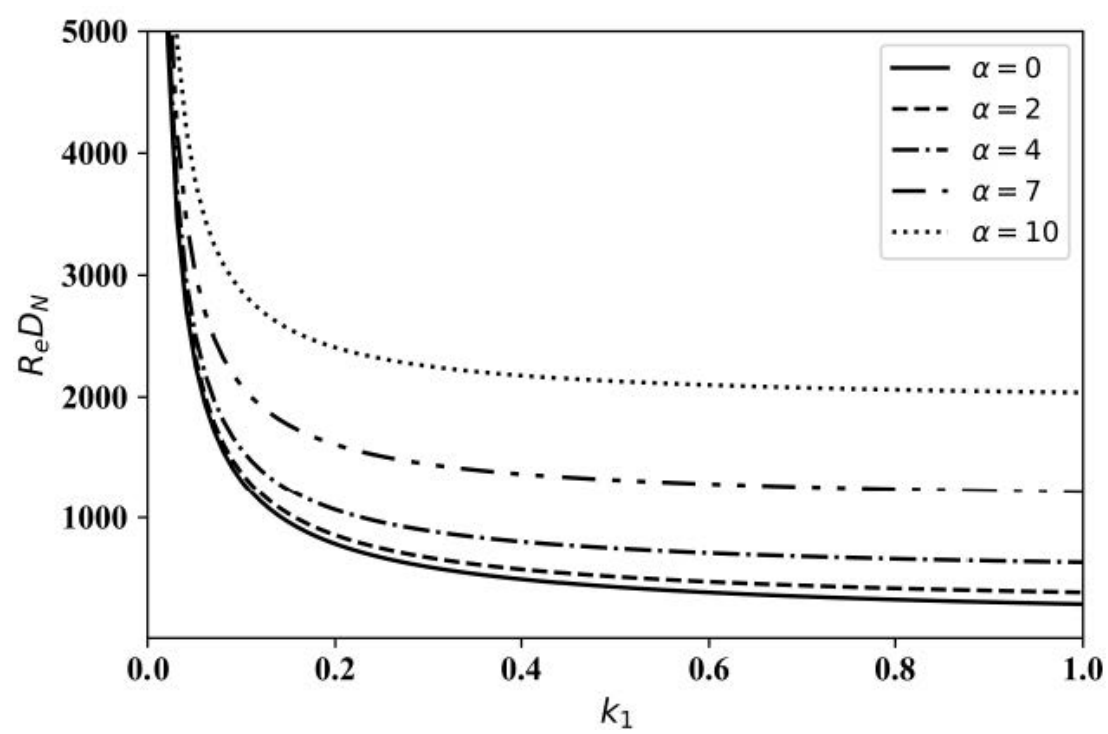

Fig. 2: Plot of $R_{e} D_{N}$ with $k_{1}$ for varying $\alpha$ with $n=0.3$ and $\chi=5$. 


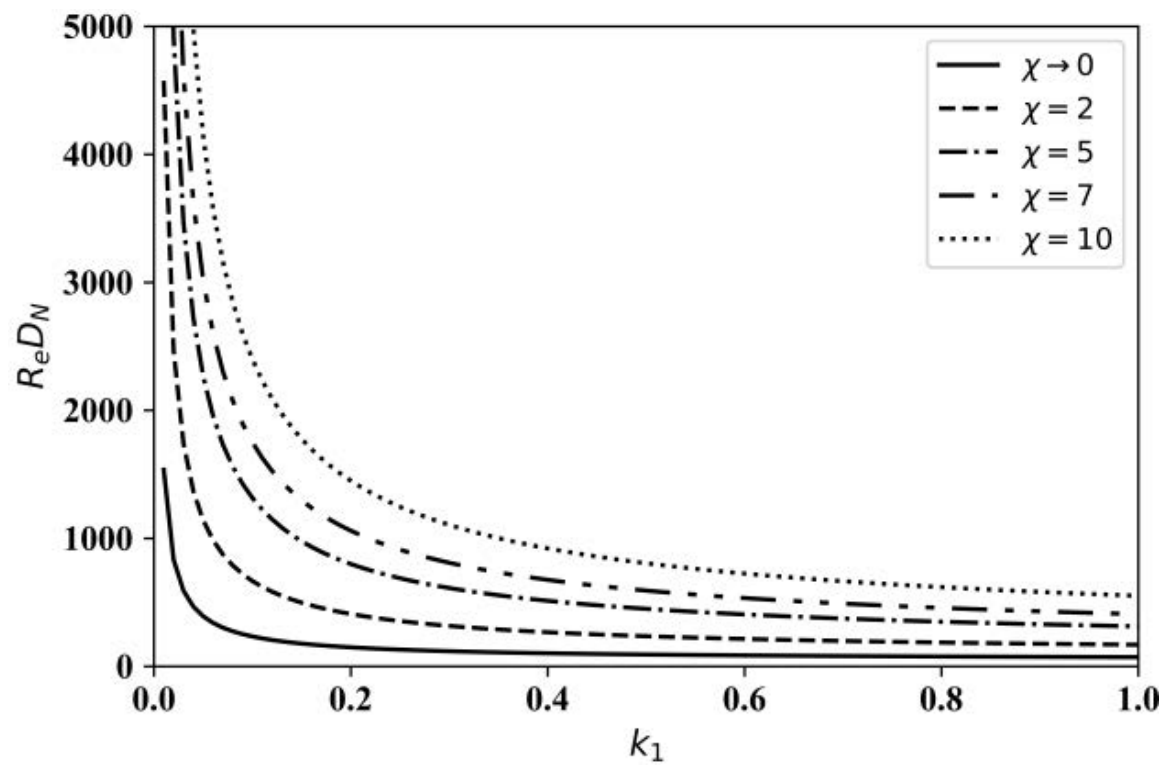

Fig. 3: Plot of $R_{e} D_{N}$ with $k_{1}$ for varying $\chi$ with $n=0.3$ and $\alpha=1$.

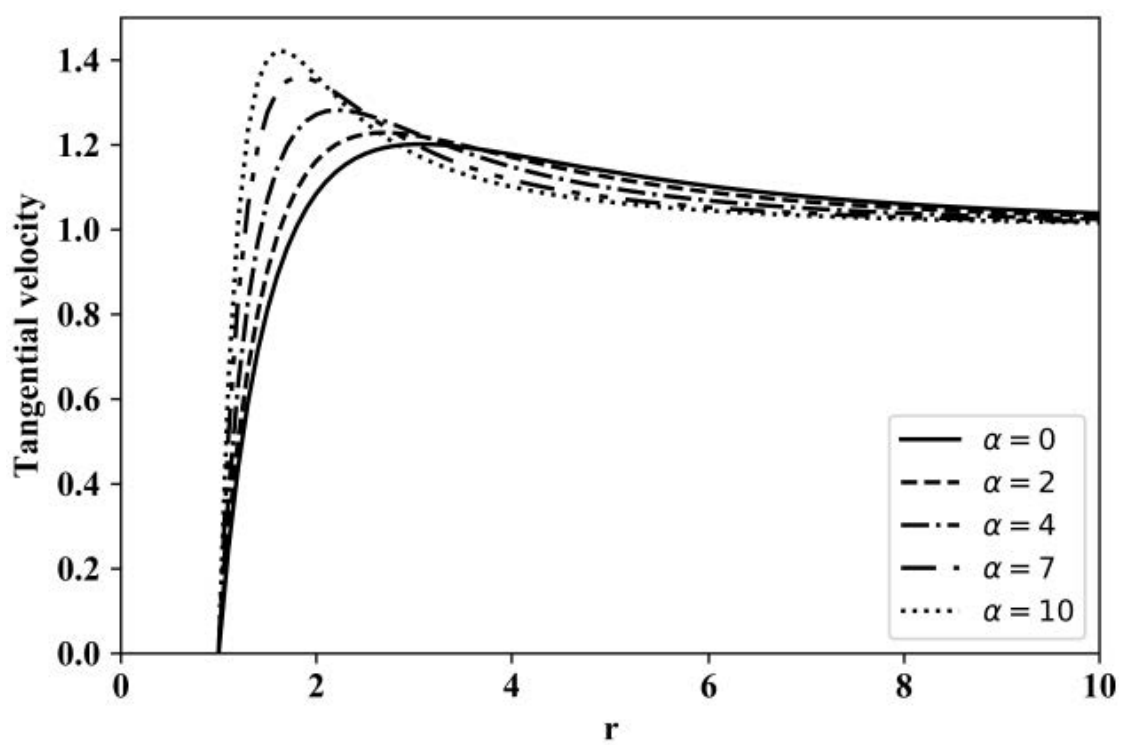

Fig. 4: Plot of tangential velocity with $r$ for varying $\alpha$ with $\chi=7, n=0.3$ and $k_{1}=1$. 


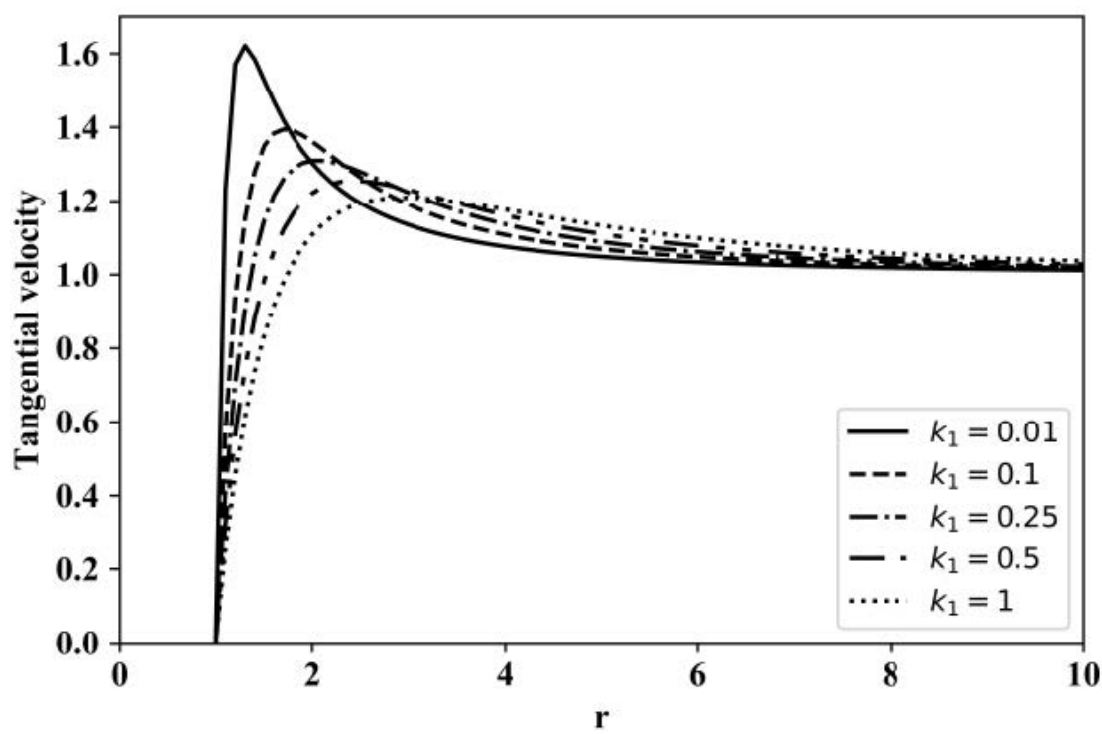

Fig. 5: Plot of tangential velocity with $r$ for varying $k_{1}$ with $n=0.3, \chi=7, \alpha=1$.

Dependence of tangential velocity profile $q_{\theta}$ on Hartmann number and permeability parameters by choosing $\chi=7, n=0.3$ and $\theta=\pi / 2$ are plotted in Fig. 4 and Fig. 5, respectively. Mentioned figures clarifies that the tangential velocity increases from zero at the wall till maximum at small distance from the wall, and asymptotes equal to unity at a distance far from the wall. Fig. 4 depicts the nature of tangential flow with increasing magnetic effect. We found that with increasing Hartmann number, the width of micropolar sublayer seems to decreases. Figure 5 demonstrates the influence of micropolarity parameter on the tangential velocity. The width of the micropolar sublayer seems to be increasing with increasing permeability.

\section{CONCLUSIONS}

This paper aims to investigate MHD effect on the flow through a cylinder implanted in a porous media. No slip of velocities and no-spin of microrotation are considered to be the boundary conditions. The present analysis yields an expression for stream function, microrotation and pressure. Influences of various parameters like Hartmann number, micropolarity parameter, permeability parameter and radius of the cylinder on the flow rate are presented in graphical form. Based on the above discussion, it is seen that drag force is highly affected by magnetic field and its presence significantly seems to increases the drag force. Our results are validated with previous results available in literature. As a future aspect of the present work, magnetic effect on 
the particles considering varying geometrical shapes implanted in a porous medium of homogenous or heterogeneous structure can be further studied through which a significant effect on the fluid flow may be obtained.

\section{REFERENCES}

[1] H.P.G. Darcy (1910) Les Fontaines Publiques de la Ville de Dijon. Proceedings of Royal Society of London Ser 83 357-369.

[2] H.C. BRINKMAN (1947) A Calculation of Viscous Force Exerted by Flowing Fluid on Dense Swarm of Particles. Applied Science Research A(1) 27-34.

[3] L. Spielman, S.L. Goren (1968) Model for Predicting Pressure Drop and Filtration Efficiency in Fibrous Media. Environment of Science and Technology 2 279-287.

[4] I. Pop, P. Cheng (1992) Flow Past a Circular Cylinder Embedded in a Porous Medium Based on the Brinkman Model. International Journal of Engineering and Science 30 257-262.

[5] J. Feng, P. Ganatos, S. Weinbaums (1998) Motion of a Sphere Near Planar Confining Boundaries in a Brinkman Medium. Journal of Fluid Mechanics 375 265-296.

[6] C.Y. WANG (2010) Darcy-Brinkman Flow with Solid Inclusion. Chemical Engineering Communication 197 261-274.

[7] D. Satya, P.K. Yadav, T. Ashish (2010) Slow Viscous Flow Through a Membrane Built up from Porous Cylindrical Particles with an Impermeable Core. Applied Mathematical Modelling 34 1329-1343.

[8] N.E. Leontev (2014) Flow Past a Cylinder and a Sphere in a Porous Medium within the Frame Work of Brinkman Equation with the Navier Boundary Condition. Fluid Dynamics 49(2) 232-237.

[9] A.C. ERINGEn (1996) Theory of Micropolar Fluids. Journal of Mathematics and Mechanics 16 1-18.

[10] A.C. ERINGEn (2001) Microcontinuum Field Theories II: Fluent Media. New York: Springer.

[11] S.K.L. RAO, P.B. RaO (1970) The Slow Stationary Flow of a Micropolar Liquid Past a Sphere. Journal of Engineering Mathematics 4 209-217.

[12] H. Hayakawa (2000) Slow Viscous Flows in Micropolar Fluids. Physical Review E 61(5) 5477-5492.

[13] M.H. Hamdan, M.T. Kamel (2011) Polar Fluid Flow Through Variable Porosity, Isotropic Porous Media. Special Topics and Review in Porous Media 2(2) 145-155.

[14] M.K. Prasad, D. SRinivasacharya (2017) Micropolar Fluid Flow Through a Cylinder and a Sphere Embedded in a Porous Medium. International Journal of Fluid Mechanics Research 44(3) 229-240.

[15] B.R. Jaiswal (2018) A Non-Newtonian Liquid Sphere Embedded in a Polar Fluid Saturated Porous Medium: Stokes Flow. Applied Mathematics and Computation 316 488-503. 
[16] R. Krishnan, P. Shukla (2019) Drag on a Fluid Sphere Embedded in a Porous Medium with Solid Core. International Journal of Fluid Mechanics Research 46219 228.

[17] D.Yu. Khanukaeva, A.N. Filippov, P.K. Yadav, A. Tiwari (2019) Creeping Flow of Micropolar Fluid Parallel to the Axis of Cylindrical Cells with Porous Layer. European Journal of Mechanics B Fluids 76 73-80.

[18] D.Yu. Khanukaeva, A.N. Filippov, P.K. Yadav, A. Tiwari (2019) Creeping Flow of Micropolar Fluid Through a Swarm of Cylindrical Cells with Porous Layer (Membrane). https://arxiv.org/pdf/1903.08415.

[19] S. Globe (1959) Laminar Steady State Magnetohydrodynamic Flow in an Annular Channel. Physics of Fluids 2 404-407.

[20] R.R. Gold (1962) Magnetohydrodynamic Pipe Flow Part-I. Journal of Fluid Mechanics 13 505-512.

[21] C.V. Raghava Rao, T.V.S. Sekhar (2000) MHD Flow Past a Circular Cylinder - a Numerical Study. Computational Mechanics 26 83-88.

[22] G.E. Geindreau, J.L. Aurialt (2002) Magnetohydrodynamic Flows in Porous Media. Journal of Fluid Mechanics 466 343-363.

[23] V.K. Verma, S. DatTa (2010) Magnetohydrodynamic Flow in a Channel with Varying Viscosity under Transverse Magnetic Field. Advance Theory of Applied Mechanics 3 53-66.

[24] A. Tiwari, S. Deo, A. Fillippov (2012) Effect of Magnetic Field on the Hydrodynamic Permeability of a Membrane. Colloid Journal 74(4) 512-522.

[25] D.V. Jayalakshmamma, P.A. Dinesh, M. Sankar (2014) Flow of Conducting Fluid on Solid core Surrounded by a Cylindrical Region in Presence of Transverse Magnetic Field. Mapana Journal of Science 13(3) 13-29.

[26] V.K. Verma, A.K. Gupta (2017) Analytical Solution of the Flow in a Composite Cylindrical Channel Partially Filled with a Porous Media in the Presence of Magnetic Field. Special Topics and Reviews in Porous Media: An International Journal 8(1) 3948.

[27] E.I. SAAD (2018) Effect of Magnetic Fields on the Motion of Porous Particles for Happel and Kuwabara Models. Journal of Porous Media 21(7) 637-664.

[28] I.A. Ans ARI, S. Deo (2018) Magnetohydrodynamic Viscous Fluid Flow Past a Porous Sphere Embedded in Another Porous Medium. Special Topics and Review in Porous Media: An International Journal 9(2) 191-200.

[29] M.K. Prasad, T. Bucha (2019) Effect of Magnetic Field on the Steady Viscous Flow Around a Semipermeable Spherical Particle. International Journal of Applied and Computational Mathematics 598.

[30] M.K. Prasad, T. Bucha (2019) Impact of Magnetic Field on Flow Past Cylindrical Shell using Cell Model. Journal of the Brazilian Society of Mechanical Science and Engineering 41320. 
[31] M.K. Prasad, T. Bucha (2019) Creeping Flow of Fluid Sphere Contained in a Spherical Envelope: Magnetic Effect. SN Applied Sciences 11594.

[32] M.K. Prasad, T. Bucha (2020) Magnetohydrodynamic Creeping Flow Around a Weakly Permeable Spherical Particle in Cell Models. Pramana Journal of Physics 94 24.

[33] F. Selimefendigil, H.F. Oztop (2018) Magnetic Field Effects on the Forced Convection of CuO-water Nanofluid Flow in a Channel with Circular Cylinders and Thermal Predictions Using ANFIS. International Journal of Mechanical Sciences. 146 9-24. 